\title{
Memory retrieval time and memory capacity of the CA3 network: Role of gamma frequency oscillations
}

\author{
Licurgo de Almeida, ${ }^{1}$ Marco Idiart, ${ }^{1,2}$ and John E. Lisman ${ }^{3,4}$ \\ ${ }^{1}$ Neuroscience Program, UFRGS, Porto Alegre, 90046-900, Brazil; ${ }^{2}$ Physics Institute, UFRGS, Porto Alegre, $91501-970$ Brazil; \\ ${ }^{3}$ Department of Biology and Volen Center for Complex Systems, Brandeis University, Waltham, Massachusetts 02545, USA
}

\begin{abstract}
The existence of recurrent synaptic connections in CA3 led to the hypothesis that CA3 is an autoassociative network similar to the Hopfield networks studied by theorists. CA3 undergoes gamma frequency periodic inhibition that prevents a persistent attractor state. This argues against the analogy to Hopfield nets, in which an attractor state can be used for working memory. However, we show that such periodic inhibition allows one cycle of recurrent excitatory activity and that this is sufficient for memory retrieval (within milliseconds). Thus, gamma oscillations are compatible with a long-term autoassociative memory function for CA3. A second goal of our work was to evaluate previous methods for estimating the memory capacity $(P)$ of CA3. We confirm the equation, $P=c / a^{2}$, where $c$ is the probability that any two cells are recurrently connected and $a$ is the fraction of cells representing a memory item. In applying this to $C A 3$, we focus on $C A 3 a$, the subregion where recurrent connections are most numerous $(c=0.2)$ and approximate randomness. We estimate that a memory item is represented by $\sim 225$ of the 70,000 neurons in CA3a $(a=0.003$ ) and that $\sim 20,000$ memory items can be stored. Our general conclusion is that the physiological and anatomical findings of $\mathrm{CA} 3 \mathrm{a}$ are consistent with an autoassociative function. The nature of the information that is associated in CA3a is discussed. We also discuss how the autoassociative properties of CA3 and the heteroassociative properties of dentate synapses (linking sequential memories) form an integrated system for the storage and recall of item sequences. The recall process generates the phase precession in dentate, CA3, and entorhinal cortex.
\end{abstract}

The CA3 region of the hippocampus has been of major interest to students of memory. This is because it is the only brain region where theory and experiment appear to have converged on how a network can store memories. The theoretical work of Hopfield (1982) posited a network in which all the neurons are connected to each other (these are termed recurrent connections). These connections are made by modifiable synapses that obey a form of the Hebb rule. According to this rule, synapses are strengthened when there is correlated presynaptic and postsynaptic activity and weakened when the activity is uncorrelated. The development of formalisms to describe such "Hopfield nets" has provided tools for analyzing the properties of memory networks. Notably, it has been possible to estimate how many memories can be stored in a distributed way by the synapses of such networks (the memory capacity).

The CA3 region has properties similar to a Hopfield net. Specifically, axons of CA3 pyramidal cells project to the dendritic layer of CA3, where they make numerous recurrent connections with other CA3 pyramidal cells. These axons innervate a substantial fraction (but not all) of the CA3 region (Ishizuka et al. 1990; $\mathrm{Li}$ et al. 1994). Moreover, the synapses that CA3 cells make on other CA3 cells are modifiable by a Hebbian form of synaptic modification (Bains et al. 1999; Pavlidis et al. 2000). These findings are in accord with the assumptions underlying the Hopfield formalism. There are thus good reasons to suspect that memory storage in the CA3 region could operate according to principles similar to those of a Hopfield net. It should perhaps be emphasized that this is not true of all hippocampal regions. For instance, the CA1 region is almost entirely lacking in recurrent connections.

\footnotetext{
${ }^{4}$ Corresponding author.
}

E-mail Lisman@Brandeis.edu; fax (781) 736-3107.

Article is online at http://www.learnmem.org/cgi/doi/10.1101//m.730207.
Several key ideas have emerged from the theoretical analysis of Hopfield nets, and these have strongly influenced how neuroscientists analyze memory networks. One important idea is that an entire memory can be recalled using only a part of the memory as a cue. A memory is represented by activity in a particular subset of cells in the network (i.e., a spatial pattern). During learning, this pattern is encoded in synapses by the learning rule. Once this occurs, this memory and other stored memories are stably encoded, even in the absence of activity. The memory recall process occurs in the following way. A memory cue is a subset of the cells that encode a memory. This cue is presented to the network and excites the corresponding cells, thereby reproducing a part of the memory. Then, through the recurrent excitatory connections, these cells excite all the cells that represent the memory, notably those that did not receive direct input from the cue. This process is called pattern completion and can be considered a memory recall process (Marr 1971). Such a process could be an important aspect of human memory; for instance, the smell of a food (the cue) may evoke the scene where it was first tasted.

A second important property of Hopfield nets is that a memory, when activated, will persist in its activity. This occurs simply because the excited cells continue to excite each other. This persistence is likely to be one mechanism of short-term memory (often termed working memory); indeed, recordings from various brain regions have revealed that reactivation of a memory can cause persistent firing even after the stimulus is removed (Wang 2001).

A third and critical aspect of Hopfield nets is their attractor property. Once a memory becomes active, it can be perturbed by external or internal factors that cause errors in firing. For instance, some cells that are part of the memory may fall silent. In some dynamical systems, such a perturbation would only get worse over time. If such a progression were to occur in a memory 
system, the memory would eventually become unrecognizable by other networks and thereby be lost. In the case of attractor networks, however, a perturbation does not cause progressive worsening; rather, the firing pattern is restored (attracted) to its original state. This repair process is possible because the memory is so redundantly encoded that the cells that remain active can reactivate the cells that stopped firing. If, on the other hand, the perturbation is strong enough, the firing pattern could potentially change drastically to another memory pattern. This abrupt (nonlinear) response is also a feature of attractor networks.

A fundamental property of any memory storage device is its capacity. One might hope that the formalisms derived for calculating memory capacity in Hopfield nets could be directly applied to CA3. However, there are many reasons that this is not straightforward. In the original Hopfield formulation, cells that fire together have the connections between them strengthened, and this is in accord with the experimental evidence for Hebbian synaptic plasticity at hippocampal synapses. However, in the Hopfield formulation and many of its extensions (Amit et al. 1987; Tsodyks and Feigel'man 1988; Treves and Rolls 1991; Curti et al. 2004), the connections between neurons that do not fire together become inhibitory. This would mean that some connections of a neuron are excitatory whereas other connections are inhibitory, a property for which there is no experimental evidence.

This lack of realism is addressed in the work of Willshaw et al. (1969) and others. In their models, synaptic connections can change their strength between zero and some excitatory value. Inhibition is present in the network, but in a separate group of cells, and is not modifiable (Golomb et al. 1990). For Willshawtype models, equations have been derived for determining memory capacity. However, there are two issues that must be addressed before these can be applied to CA3.

The first issue concerns the fact that CA3 does not seem to demonstrate persistent firing. This is generally considered a fundamental property of Hopfield nets (and of the model analyzed by Willshaw). The experimental test for persistence is to present a cue and to determine whether the evoked activity persists after the cue is removed. Although many brain regions have such "working memory" properties, there have been no reports of clear persistent activity in the hippocampus. This has been most extensively explored in the context of trace conditioning, a form of learning for which the hippocampus is required. In this paradigm, there is a few-second interval between the end of the conditioned stimulus and the unconditioned stimulus, and so the brain must somehow retain a memory trace of the conditioned stimulus to form the needed association. It was suspected that persistent firing in the hippocampus might form such a trace, but experiments have not shown it to be there (for reviews, see Rodriguez and Levy 2001; Levy et al. 2005).

Indeed, from a biophysical standpoint, it is unlikely that CA3 could demonstrate persistent activity. Analysis of the requirements for persistent activity indicates that special mechanisms must be in place to prevent synchronized inhibition or to allow persistent activity even in the presence of synchronized inhibition (Wang 1999). This is because synchronized inhibition may cause such a large fraction of cells to stop firing that there is insufficient residual activity to restore the memory after inhibition wanes. Studies of field potentials in CA3 show strong gamma-frequency oscillations that are indicative of synchronized inhibition (Csicsvari et al. 2003). One possible mechanism for reinitiation is due to current through slow NMDA channels that were activated on the previous gamma cycle. However, if the intervening inhibition has hyperpolarized the neuron, the NMDA channels are blocked by $\mathrm{Mg}^{2+}$, and could be reopened only if some spontaneous depolarizing noise occurred. Such conditions are not impossible, but unlikely.
An altogether different argument that CA3 might show persistent activity is that it acts as an integrator network, a type of network that relies on persistent activity. The notion that CA3 acts as an integrator derives from the study of CA3 place fields, which indeed show integrator properties. However, it now seems clear that these properties are not computed in the hippocampus itself, but are derived from upstream cortical networks (McNaughton et al. 2006).

These findings raise the question of whether the ideas developed about Hopfield-type networks can be meaningfully applied to the CA3 network. Of specific interest to us is whether the relationship between memory capacity and the effective network connectivity derived by Willshaw is applicable to oscillatory networks like CA3. A second issue relates to the structure of CA3 connectivity itself. Of all the known brain networks, CA3 most closely approximates the all-to-all architecture that Hopfield analyzed. However, it is clear that the all-to-all assumption is not valid in CA3; rather, the connections are sparse. This has been appreciated for some time and has been taken into consideration by theoretical work that assumes the connections are sparse and random. However, the assumption of randomness is not strictly correct; there is a patterning of the recurrent axons such that from any given part of CA3 they project more to some parts of CA3 than to others (Ishizuka et al. 1990; Li et al. 1994). This non-randomness of connectivity has not been taken into consideration in previous theoretical work. It could mean that the entire analogy to Hopfield networks is fundamentally flawed. We have analyzed this issue and concluded that the analogy remains useful; a crude way of taking the non-randomness into consideration is suggested.

A major goal of our work has been to estimate the memory capacity of the CA3 network. Because we found the Willshaw formula applicable, our task was reduced to estimating the two key parameters in the formula, the sparseness of recurrent synaptic connections and sparseness of memory representations. Since the number of cells in the network is known, we could calculate sparseness of representation by estimating the size of the neuronal ensemble that represents a memory. We have used several strategies to estimate this fundamental number.

In the Discussion, we review our previous theoretical work concerning how CA3 and the dentate gyrus function together to store memory sequences, a central requirement of episodic memory. This class of models is capable of explaining how a phenomenon called the phase precession $\left(\mathrm{O}^{\prime}\right.$ Keefe and Recce 1993) is generated through the interactions of dentate and CA3. This phenomenon is of considerable importance because it is likely to represent the recall of memory sequences. The autoassociative function of CA3 can thus be placed into a very specific computational, anatomical, and physiological context. However, recent work observing the phase precession in the entorhinal cortex (Hafting et al. 2007) raises the possibility that the precession observed in dentate/CA3 is simply inherited from cortex. We address this issue in the Discussion. Our analysis suggests that the phase precession in the dentate/CA3 is likely to be different from that in the entorhinal cortex and is therefore likely to be generated in dentate/CA3, as postulated in our models (Lisman and Talamini 2005).

\section{Results}

General operation of networks with recurrent excitation and recurrent inhibition: Fast memory recall within a gamma cycle

Gamma frequency oscillations are evident in the CA3 region (Csicsvari et al. 2003) and thus must be taken into consideration 
when analyzing the region as a memory network. The mechanistic framework for the generation of gamma oscillations has been established through a broad range of experimental work. According to this framework, gamma is generated by synchronized firing in pyramidal cells (for review, see Bartos et al. 2007). This provides convergent input to interneurons, which in turn provide rapid feedback inhibition to the pyramidal cells. This rapid feedback inhibition insures that their firing occurs within a small temporal window. As the inhibition slowly wanes, the most excitable pyramidal cells reach threshold and fire, thereby initiating the next gamma cycle. In the hippocampus, the key interneuron subtype involved in gamma oscillations is the basket cells (Hájos et al. 2004). For a review of interneuron properties, see Somogyi and Klausberger (2005).

Previous theoretical work has shown that presence of gamma is not incompatible with memory function (Jensen and Lisman 1996a,b,c; Jensen et al. 1996). In particular, the critical recall process of pattern completion can be performed if recurrent excitatory connections are present. The reason for this compatibility stems from the fact that recurrent excitation is monosynaptic, whereas recurrent (feedback) inhibition is disynaptic, and therefore slower. The differences in latency of feedback excitation and feedback inhibition have been directly demonstrated by paired recordings (Miles 1990). This difference creates a short time window in which recurrent excitation can perform pattern completion, as illustrated in Figure 1. What has been simulated here is a simplified network of integrate-and-fire neurons. They are presented with a cue (a partial memory). About 5 msec after the excited cells fire, the recurrent excitatory connections cause firing in the other cells of the same memory. Shortly thereafter, inhibition builds up to a high level and firing ceases. Thus, all cell firing occurs within a 5-msec window that is a relatively small fraction of the overall gamma cycle (if there were continued excitation, the next period of firing would occur after $\sim 20$ msec because of the decay of inhibition). One can see from this example that a network based on recurrent inhibition and
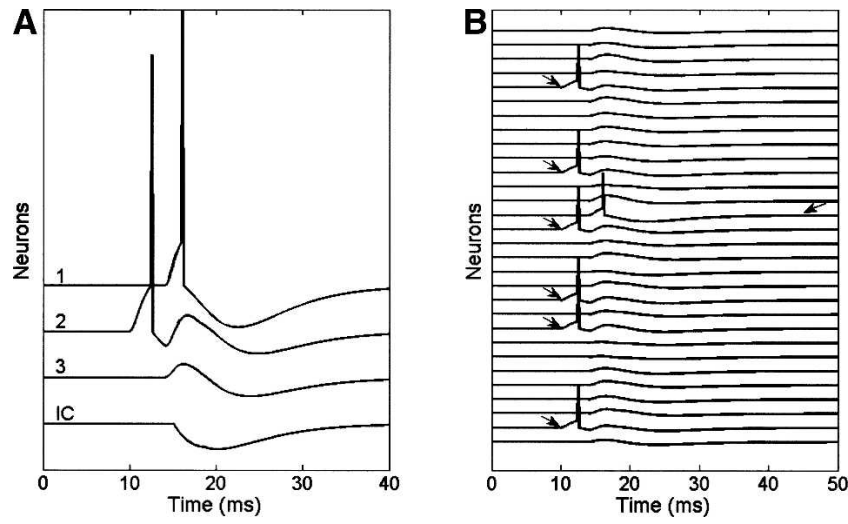

Figure 1. Memory recall in a single gamma cycle. At $t=10 \mathrm{msec}$, the network is presented with a memory cue that excites six of the seven cells that represent a memory. $(A)$ Intracellular voltage and its component parts: Trace 2 shows a cell directly excited by the cue. Trace 1 shows the recall process in a cell that is part of the memory, but that was not directly excited by the cue. It receives an EPSP from the cell that was excited by the cue and fires. All cells are affected by the feedback inhibition (IC), which is slightly delayed relative to feedback excitation. This inhibition "resets" the network. Trace 3 shows the feedback excitation and inhibition in a cell that is not part of the memory and that does not receive enough excitation to fire. (B) The activity of all neurons in the network (30 neurons). The memory is represented by seven neurons, but the external input is incomplete, and only six neurons were excited (arrows at left). The recurrent connections cause memory recall (pattern completion) by firing the cell marked with the arrow at the right side. excitation (1) will generate gamma oscillations, (2) has synchronized firing of pyramidal cells in a small fraction of the gamma cycle, and (3) is capable, in a single feedback cycle, of performing memory recall (pattern completion). For additional information about the operation of such memory networks see Jensen et al. (1996).

\section{Estimating the memory capacity of CA3}

The calculation of memory capacity of a network requires an analytical framework. Several such frameworks for the study of associative memory have been proposed. These differ in how individual cell are represented (binary firing, firing-rate, integrate-and-fire, or a more complex Hodgkin-Huxley), how the synaptic plasticity that underlies learning is implemented, and how the actual architecture of the network is implemented. Despite these differences, there is agreement (at least to a first approximation) that the parameters that determine memory capacity are basically the size of the network, $N$, its connectivity, $c$ (the average fraction of the cells connected to any given neuron), the range of the synaptic strength, and the sparseness ratio of neural coding, $a$ (the average fraction of active neurons in the network during a single memory item).

In Hopfield-type models, the presynaptic and postsynaptic activities are measured relative to the average activity of the network, and the correlation rules lead to positive and negative synapses. Another rather artificial feature is that synapses between inactive neurons can be strengthened. For these models, a formula for memory capacity, $P$, was derived (Tsodyks and Feigel'man 1988; Buhmann et al. 1989) for binary neurons and fully connected networks. An extension of this formula for graded response neurons and arbitrary connectivity was proposed by Treves and Rolls (1991) as

$$
P=\frac{k c_{\mathrm{m}} N}{a \log \left(\frac{1}{a}\right)}
$$

where $c_{\mathrm{m}}$ is the connectivity of the modifiable synapses, $N$ is the size of the network, $k$ is a constant between 0.2 and 0.3 , and $a$ is a generalization of the sparseness ratio for firing rate neurons. Even though different aspects of this equation were derived previously by a number of other investigators, we will refer to Equation 1 as Treves' formula, since it is in Treves and Rolls (1991) that this more general form is proposed.

In another class of models (Willshaw et al. 1969), the synapses are considered to be only positive or null (silent): positive in the case where neurons fire together in a pattern and null if this never occurs. For Willshaw's models, the estimated storage is smaller and was derived in Golomb et al. (1990) for random sparse patterns. The storage limits depend of the details of how inhibition is done, but a very general expression can be found to relate connectivity and the number of memories in the network. This expression is

$$
P=\frac{c}{a^{2}}
$$

where $c$ is the connectivity of the network due to the storage of the patterns. Observe that $c$ differs from $c_{\mathrm{m}}$. In the context of Hopfield networks $c_{\mathrm{m}}=1$ for an all-to-all network or could be less for a low connectivity network, but $c_{\mathrm{m}}$ will be an external parameter always independent of the memory storage. In a Willshaw network, on the other hand, $c$ reflects the memory storage, in the sense that $c$ increases as we store more memories in the network. So Equation 2 does not, in fact, represent an expression for a storage limit; rather, it represents a relationship between connectivity and storage. If, however, we add the hy- 
pothesis that what is experimentally observed in CA3 is already a connectivity that reflects its maximal storage capacity, Equation 2 can be used to derive the number of memories stored there. We will refer to Equation 2 as Willshaw's formula, due to its broad applicability to Willshaw-like models.

\section{Numerically testing equations for memory capacity}

Our goal in this section was to test for memory capacity under conditions that approximate fast recall (i.e., within a single gamma cycle). This may allow us to determine the validity of the different analytical expressions for memory capacity (Equations $1,2)$.

We selected each memory by randomly choosing $A$ cells out of the total of $N$ cells. We selected a total of $p$ memories. These memories were incorporated into the synaptic matrix $W$ using the synaptic plasticity rules proposed by Jensen et al. (1996) (see Appendix), which can be considered to be of Willshaw's type. Later, the synaptic matrix was tested for retrieval of all patterns. In order to do that, we calculate the recurrent input to the network upon presentation of the memorized binary pattern $b^{\mu}$,

$$
I^{\mu}=W \times b^{\mu}
$$

where the right side is the dot product between the synaptic matrix and the activity vector, and $I^{\mu}$ is the resulting vector of recurrent inputs. The pattern $\mu$ is considered successfully stored if the lowest input to any neuron that is part of the pattern is larger than the maximal input to any neuron that is not part of the pattern.

$$
\min \left(I_{\text {active }}^{\mu}\right)>\max \left(I_{\text {inactive }}^{\mu}\right)
$$

If this is true, there exists a threshold, or a value for feedback inhibition, that can successfully separate the neurons that are active in a pattern (signal) from neurons that receive inputs but should be silent in that pattern (noise or an overlapping memory), and no false positives or false negatives are generated. If any neuron in any of the $p$ patterns failed to satisfy that condition, we considered that the storage failed. We assumed that if the synaptic matrix passed this requirement, it would pass a more realistic test with integrate-and-fire neurons, as we show later. We varied $\mathrm{p}$ until we found a value where the test failed. This value of $p$ was taken to approximate the maximal storage capacity, $P$.

We used two different procedures for progressively adding memories. In the first, we added random patterns to the synaptic matrix and stopped when the limit was reached. In the second, we followed more or less the same procedure, adding random patterns, but when a pattern led to a failure in storage, we discarded it and tried a new one. This is a slow procedure, and we tried only a fraction of all possible patterns of $A$ active among $N$ neurons before concluding that the limit was attained. Because the selection process in the second procedure introduced a nonrandom aspect, we repeated the whole process several times in order to obtain a useful (average) characterization. With the second procedure, we found memory capacity to be about three times larger than with the first (see below).

Figure 2 displays the simulation results for several network sizes as well as different activity fractions. The points are well approximated by Willshaw's formula. It is important to point out that Equation 2 is exact for nonoverlapping memories, and it is approximately correct for random overlapping memories (Golomb et al. 1990). We show here that it is also correct for selected patterns, indicating that this expression is quite general.

Treves' expression is more difficult to apply to these data, since it was derived for Hopfield-like models (where synapses between inactive neurons can be strengthened), making the con-

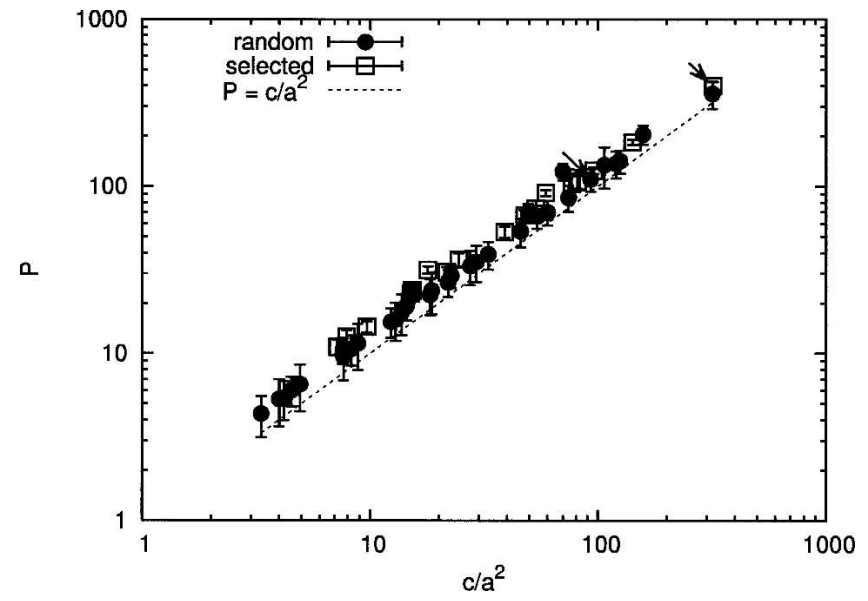

Figure 2. Measurements of storage capacity. Calculations were made for different network size (from $N=10$ to 1000) and sparseness ratio (from $a=0.01$ to 0.3 ). (Filled circles) Random patterns, (empty squares) selected patterns for maximal storage. On average, when patterns are selected, it is possible to store three times more patterns for the same sparseness ratio and network size. (Arrows) $N=500$ and $A=15$ $(a=0.03)$, (dashed line) predicted relationship for Willshaw's network.

nectivity not directly related with memory load, but rather to some predefined architecture. If we replace $c_{\mathrm{m}}$ by $c$, the resulting plot (Fig. 3, left) is scattered, indicating that formula is not a good predictor of storage. If, on the other hand, we consider $c_{\mathrm{m}}=1$, since in our numerical simulation in principle all synapses are plastic, the resulting plot (Fig. 3, right) indicates that Treves' formula can be used as an upper limit, but the effective connectivity is no longer a parameter available for estimation of capacity.

Use of an integrate-and-fire model to test the memory capacity predicted by the Willshaw equation

We next sought to test the Willshaw-like learning rule in the context of an actual simulated network. We constructed a network of integrate-and-fire neurons connected by recurrent excitatory synapses and feedback inhibition. The details of the model are presented in the Appendix. Figure 4 shows examples of retrieval of memory (pattern completion) in a network where 13 memories have been previously stored in the network. This matrix was found by the procedure where patterns are selected for maximal storage, leading to the matrix of synaptic weights shown in Figure 4D. The question we sought to address is whether a network of integrate-and-fire neurons loaded with these memories could correctly recall them.

Figure 4A displays the network response to the stimulation of a memory pattern (memory 1 ). Figure $4 \mathrm{~B}$ displays the pattern completion process after the network is presented with an incomplete pattern (the cue). Figure $4 \mathrm{C}$ shows that a different memory (memory 2), which overlaps with memory 1 , also can be completed when presented with an appropriate cue.

A concern in this analysis is the robustness of the parameters. If the inhibition parameter had to be fine-tuned, that would indicate a lack of robustness. To study robustness, we tested the same matrix for all stored memories, but varied the inhibition delay $\left(t_{\text {delayGABA }}\right)$ and its amplitude $\left(A_{\mathrm{GABA}}\right)$. We provided the complete pattern and tested for incorrect responses, defined by the firing of any neuron that was not part of the memory or the firing of any memory neuron more than once. Figure 5 shows the range of parameters tested and the response for each configuration. The white area represents the range of parameters where the network responds correctly to all memo- 

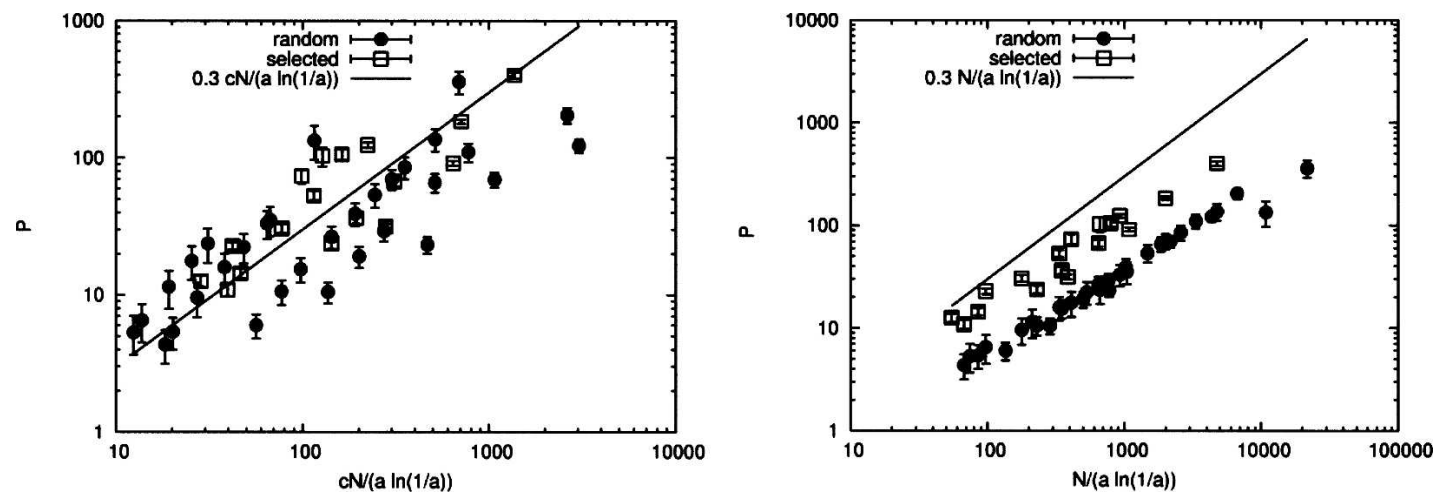

Figure 3. Comparison of simulated memory capacity to Treves' formulas. (Filled circles) Random patterns, (empty squares) selected patterns for maximal storage. (Left) Here, the resulting connectivity $(c)$ of the matrix is used in place of $c_{\mathrm{m}}$. The points for the different simulations appear scattered, indicating that Equation 1 doesn't predict the simulation data. (Right) For $c_{m}=1$, the points for random patterns separate from the ones of selected patterns. Equation 1 seems to fit the results for random patterns (but only for low values of $k$ but doesn't fit the data for selected patterns. Treves' formula (line in both panels) with $c_{\mathrm{m}}=1$ seems to be an upper bound for capacity in our simulations.

ries, and the dark area represents the space of parameters where the responses were incorrect for all memories. This figure shows that the network works correctly over a substantial parameter range. We conclude that the memory capacity, as estimated by the Willshaw formula, can be achieved by realistic networks.

\section{CA3 is not a uniform network with random recurrent connections}

The recurrent connections of CA3 are not completely random. Pyramidal cells closest to the dentate (CA3c) project strongly to CA1 and have relatively few recurrent connections with CA3 cells. On the other hand, the CA3a section that is closest to CA1 has very strong recurrent connections with other cells in CA3a and relatively few connections with CA1 (Ishizuka et al. 1990; Li et al. 1994). The strong recurrents probably account for why the $\mathrm{CA} 3 \mathrm{a} / \mathrm{b}$ region is the site of initiation of epileptiform activity (Colom and Saggau 1994; Dzhala and Staley 2003). These specializations of CA3 subregions have been ignored in previous computational studies. Dealing quantitatively with these specializations is complicated because the differences are tendencies rather than being absolute. As a crude simplification, we will assume that they are. Thus, we assume that CA3a is the best model for a strongly interconnected associative network. As a corollary, CA3c can be considered an output structure for CA3 to CA1 (see Discussion) and CA3b as intermediate-type structure. We will therefore analyze hippocampal memory capacity using the approximation that the critical autoassociative function occurs in CA3a.

\section{Estimate of sparseness of connectivity}

Recordings from cell pairs in CA3 (Miles 1990) in the acute slice preparation indicate that cells have a rather low probability of being connected (0.05). However, this must be considered a lower limit because of the possibility that connections were severed by cutting the slice and because no tests were conducted to detect silent synapses (depolarization might have a revealed NMDAR-mediated component). Furthermore CA3a was not specifically tested.

A higher estimate of connectivity is derived from anatomical considerations. The CA3a subregion contains the highest density of recurrent collateral axons ( $\mathrm{Li}$ et al. 1994). Such axons can have 40,000 varicosities (Wittner et al. 2006) and each can potentially contact a pyramidal cell. All of CA3 contains 200,000 cells (Rapp and Gallagher 1996), 70,000 of which would be in CA3a. Thus, from this perspective, if all varicosities were in CA3a (an upper limit), it would be possible for a CA3a cell to send a signal to $\sim 50 \%$ of CA3a cells.
Another perspective is to look at the capacity of CA3 cells to be information receivers. We assume that there are $\sim 30,000$ spines in the dendritic region that contains associative synapses (this assumption is based on measurements in CA1; Bannister and Larkman 1995). Thus, CA3 cells could potentially receive input from about half of the cells in CA3a. This is again an upper limit. Taking a midpoint between upper and lower limits described above, we will assume that connectivity is on the order of $20 \%$ in CA3a.

\section{Estimate of ensemble size (sparseness of coding)}

An ensemble is a group of cells that fire together. In the context of place fields, "together" means that they fire in the same position in space and with the same theta phase. Work on place cell ensembles has identified groups that fire together at a particular phase of theta. Different ensembles, with slightly different positions of maximal firing, fire at slightly different phases. Such analysis indicates that the minimal spatial separation that corresponds to a detectable difference in theta phase is on the order of few centimeters (Dragoi and Buzsáki 2006). A similar conclusion is reached using a formal mathematical method for position reconstruction from multiple place fields, which indicates that the rat's position is definable with an accuracy of a few centimeters (Jensen and Lisman 2000). Thus, if $100 \mathrm{~cm}$ (the size of typical linear track) is uniformly represented by different ensembles with 2 -cm precision, there will be $\sim 50$ ensembles. Of the total cells, it is generally estimated that about half the pyramidal cells encode place in a given environment. Thus, if we restrict ourselves to the dorsal half of CA3a (35,000 cells) where place cells are numerous, there would be $\sim 17,000$ place cells and the ensemble size would be $\sim 300$. The value of 0.5 for probability of a cell being a place cell would be lower if there are cells that cannot be detected at all by spike classification methods, as is probably the case (Henze et al. 2000).

An alternative way of estimating ensemble size is to ask what is required to fire a neuron; for pattern completion, it must be possible for the memory cells that did receive the cue (which may be most of them and thus constitute an ensemble), to fire a cell that did not receive the cue. The number of effective synaptic inputs required to fire a spike in a hippocampal pyramidal cells has been investigated in several studies. If the synaptic inputs are clustered on a dendritic branch, Gasparini et al. (2004) and Gasparini and Magee (2006) estimate that 50 inputs are required. If the active inputs are random in the dendritic field, as we will assume, then Gasparini et al. (2004) estimate that 150 inputs are 

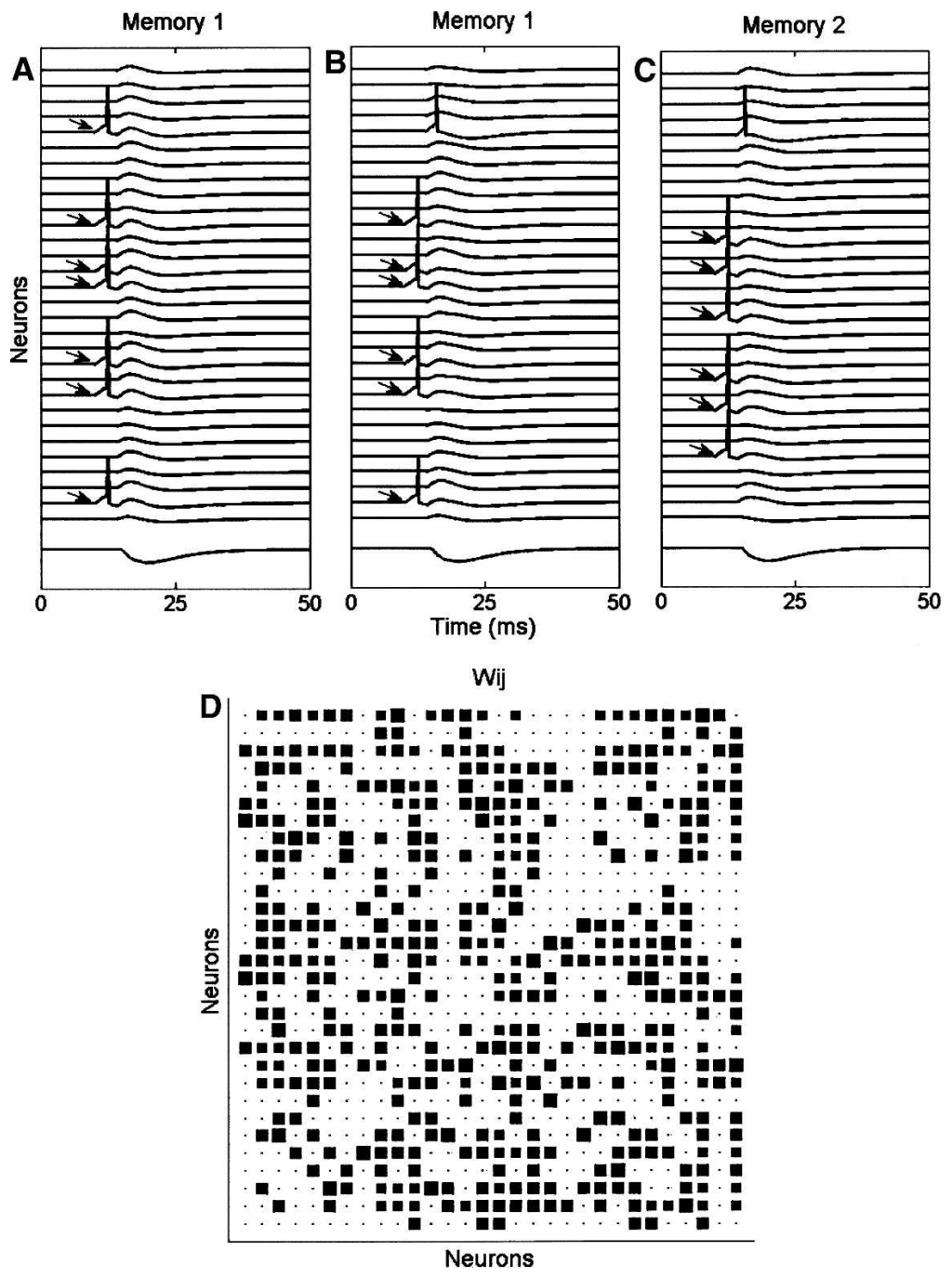

Figure 4. Testing Willshaw's synaptic matrix in an integrate-and-fire network. $(A)$ Network response to a complete memory pattern (seven neurons). (B) Response to an incomplete version of the same memory. The complete memory was recalled. (C) The recall of a different memory that overlaps with that in $B$. (Arrows) Neurons stimulated by an external input, (lines in the bottom) inhibitory currents. For these simulations, the recurrent excitatory delay is $1.5 \mathrm{msec}$ and the current is $1600 \mathrm{pA}$; the inhibitory delay is $2.5 \mathrm{msec}$ and the current is $-180 \mathrm{pA}$. For other details about parameters and functions describing the currents, see Appendix. (D) Synaptic matrix ( $\mathrm{W}_{\mathrm{ii}}$ ): Each connection between two cells is represented by a square; the size of the square denotes the synaptic strength of the connection between two cells.

required (these must be highly synchronized and there must be no inhibition). However, another group has estimated a considerably smaller number (15-30) under these conditions (Otmakhov et al. 1993). It is important to recall that synapses release transmitter probabilistically. Taking the midpoint between the estimates of needed release events and taking the average probability of release as 0.3 (Malinow et al. 1994; Oertner et al. 2002), the number of presynaptic cells that would have to be active in order to fire the postsynaptic cell is $\sim 150$. Since inhibition is present, this number must be considered a lower limit.

Taking a midpoint between the upper and lower limits described above, we will assume that within CA3a, the ensemble size is $A=225$ (of 70,000 cells). This translates into a sparseness of coding of $a=0.003$. We also assume a sparseness of connectivity of $20 \%(c=0.2)$. These numbers lead to an estimation of 20,000 memories for CA3a if we use Willshaw's equation (Equation 2). Treves' formula (Equation 1), which we do not think is altogether valid, would predict 200,000 memories for the same parameters.

\section{Discussion}

We have analyzed the CA3 region and considered its analogy to Hopfield networks (and its variants) that have been used by theorists to understand the basic properties of associative memory. We have enumerated some important differences: (1) CA3 does not appear to show the persistent activity characteristic of attractor networks, (2) CA3 has gamma frequency oscillations that are not presented in mathematical models of attractor networks, and (3) CA3 recurrent connections are not random, as assumed in previous theoretical models.

Despite these differences, we conclude that the analogy still holds, with certain qualifications. Notably, the ideas of pattern completion that is critical for memory recall can be executed by recurrent excitatory connections, even though oscillatory inhibition prevents the persistent firing that is generally considered a hallmark of Hopfield networks. Thus, we argue that the memory completion property and the persistent firing property of Hopfield networks are separable. The non-random aspect of the recurrent connections does indeed seem to violate the spirit of the Hopfield net architecture, but within CA3a, a subregion of CA3, the assumption of random, dense recurrents appears to be a reasonable approximation.

\section{Memory capacity of CA3}

It was of interest to determine the memory capacity of CA3a. We analyzed whether the equation for memory capacity derived by Willshaw (Equation 2) is applicable to oscillatory brain networks and conclude that it is. We have estimated the two critical parameters of the Willshaw equation, the sparseness of coding (the number of neurons that fire in the ensemble that represents a memory) and the sparseness of connectivity among the cells of CA3a. With these estimates, we calculate that the 70,000 cells in CA3a can store $\sim 20,000$ memory items. Although words are not stored in the hippocampus, the fact that the average educated person knows $\sim 10,000$ words (http://thelinguist.blogs.com) gives some perspective on this number. We will return later to what actually constitutes a memory item in CA3. The estimate of 20,000 memories is of course very approximate. Because capacity depends inversely on the square of ensemble size, capacity is particularly sensitive to this parameter. We estimate ensemble size at $\sim 225$, but this could easily be off by a factor of two in either direction, leading to a possible range of 5000-100,000 memories.

\section{High-speed memory recall}

As shown here, and in previous work on memory completion in oscillatory recurrent networks (Jensen and Lisman 1996b), 


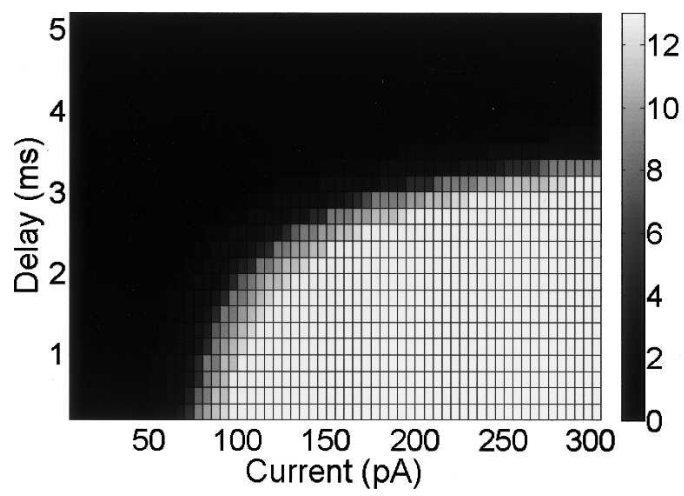

Figure 5. Test of the range of inhibition parameters that produces correct pattern retrieval. The color range represents the number of memories retrieved correctly for each set of parameters: (White area) Parameters where the integrate-and-fire network worked correctly for all 13 memories stored in the synaptic matrix; (dark area) parameters where the network could not provide a correct response to any of the 13 memories. All other shade levels represent parameters where the network worked correctly for some memories stored in the matrix but not for all. Here, the number of neurons is 30 and each memory is represented by seven neurons. The recurrent excitatory current is $1600 \mathrm{pA}$ and the delay is $1.5 \mathrm{msec}$; inhibitory current varied between 0 and $-300 \mathrm{pA}$, and the delay varied between 0 and $5 \mathrm{msec}$.

memory recall can be very rapid, in $\sim 5 \mathrm{msec}$. This duration is due to the time that it takes the cells excited by a cue to excite the cells that are part of that memory, but not directly excited by the cue. Measurements of transmission in the excitatory recurrent axons show that the time required for the action potential to reach the target cell, for synaptic transmission, and for the firing of an action potential in the target cells is on the order of $5 \mathrm{msec}$ (Miles 1990). Thus, pattern completion can occur within a 5-msec time period. Furthermore, this completion process, while perhaps not perfect (we have not considered aspects of noise and false positives), produces useful pattern completion in a single cycle or recurrent activity.

Although it is generally thought that memory retrieval in a Hopfield network requires many cycles, recent analysis indicates that much of retrieval actually occurs in the first cycle. The contrary view that many cycles are required emerged from numerical studies (Frolov and Husek 2000), indicating that the average convergence time for the traditional Hopfield model (Hopfield 1982) for non-sparse patterns (50\% active neurons) depends as a power law on the size of the network. Such estimates, when applied to CA3 network size, would indicate convergence after 8-40 cycles (Risau-Gusman and Idiart 2005), depending on the memory load. That would imply a convergence time in the range of 20 $100 \mathrm{msec}$, if we assume a minimal time of $2-3 \mathrm{msec}$ per cycle. This is quite a long time in terms of brain computations. However, recent numerical studies have analyzed the convergence process further and found that it actually occurs in two phases (Risau-Gusman and Idiart 2005): an initial rapid phase of one or two time steps that occurs in a time that is independent of network size, and a second slow phase that is dependent on network size. The important finding is that the first phase does virtually the entire process of memory completion and that the improvement produced by the second phase is miniscule. Therefore, for all practical purposes, the convergence time can be considered to occur in one to two cycles, even for large Hopfield networks. Fast autoassociative recall was also demonstrated in simulations of integrate-and-fire neurons by Battaglia and Treves (1998), confirming the mathematical analysis of Treves (1993). Both papers show that the primary determinant of the rapid pattern comple- tion time scale is the inactivation time of excitatory conductances. We conclude that both abstract Hopfield networks and real networks based on gamma oscillations can be expected to perform quite accurate memory completion within a single recurrent cycle.

\section{CA3: Autoassociation versus heteroassocation}

There have been some suggestions (Abbott and Blum 1996; Jensen and Lisman 1996a; Lisman 1999; Levy et al. 2005) that CA3 may not be an autoassociative network at all, but rather a heteroassociatve network specialized (by itself) for sequence storage and recall. The basis of these suggestions has to do with properties of the learning rule that governs hippocampal synapses. A requirement of autoassociation is the formation of symmetrical connections between cells that are part of the same memory. However, there is a fundamental difficulty with achieving this at hippocampal synapses because the learning rule is asymmetric in time; LTP occurs if a presynaptic action potential precedes the postsynaptic action potential, but not vice versa. This asymmetry results simply from the properties of the NMDA channels that trigger LTP. As has been incorporated into several models (Abbott and Blum 1996; Jensen and Lisman 1996a; Lisman 1999; Levy et al. 2005), this asymmetry is exactly what is required for learning memory sequences, and for this reason CA3 may be considered a heteroassociative network linking memories of events that occurred at different times. If this is true, then CA3 is simply not an autoassociative network, as is assumed in most theoretical work (Marr 1971; Kunec et al. 2005; Rolls and Kesner 2006).

In the next section, we will review a proposal for how networks with asymmetrical connections can in fact perform autoassociation, provided cells fire in bursts. First, however, it is useful to review experimental evidence that the CA3 region is indeed performing an autoassociative function. Nakazawa et al. (2002) tested and confirmed the idea of pattern completion by generating and analyzing genetically altered mice where the NMDA receptor gene was knocked out of CA3 pyramidal cells. These mutant mice normally acquired and retrieved spatial reference memory in the Morris water maze, but they were unable to retrieve this memory when presented with a partial cue. Similar results were obtained by Gold and Kesner (2005) with neurotoxic injections into CA3 of rats trained to find food based on external cues. When part of the cues was removed, control animals displayed excellent pattern completion across all reductions in the availability of cues, whereas rats with CA3 lesions were impaired in pattern completion, as indicated by a linear increase in errors as the number of available cues was reduced. Results from Lee et al. (2004), Leutgeb et al. (2004), and Vazdarjanova and Guzowski (2004) also showed that CA3 has critical properties of autoassociational networks. Lee et al. (2004) monitored CA3 and CA1 activity from rats running in a circular environment with distinct, familiar cues on the walls and on the surface of the track. In each experiment, standard sessions were interleaved by mismatch sessions where the cues on the track and the cues on the wall were rotated to opposite sides. For small mismatches $\left(<45^{\circ}\right)$, both CA1 and CA3 displayed coherent representations that were similar to those of the original cue configuration. However, when the mismatch amounts were $>45^{\circ}$, the CA1 representation lost its coherence; in contrast, the $\mathrm{CA} 3$ representation was more coherent between the familiar environment and mismatch environments. In other work, Vazdarjanova and Guzowski (2004) used imaging of immediate-early gene (IEG) expression as a measure of neuronal activity. In this work, the investigators examined the responses of CA3 and CA1 ensembles in rats exposed sequentially to two environments that could be identical, 
slightly similar, or completely different. The results showed that when the animals were presented with small changes, ensembles in CA3 had a higher degree of overlap when compared with CA1, similarly to Lee et al. (2004). However, when the rats were exposed to two completely different environments, the ensemble representations were highly orthogonal in CA3. The same behavior for completely different environments was observed by Leutgeb et al. (2004), where the rats were sequentially tested in enclosures with different shapes and sizes while CA3 and CA1 were monitored. These two behaviors, while in apparent conflict, are perfectly in accord with the assumption that attractor networks respond nonlinearly to input patterns.

Taken together, these neurophysiological and behavioral studies provide support for the idea that CA3 performs autoassociation and, by implication, not heteroassociation. It is therefore important to consider what synaptic plasticity mechanisms would make autoassociation possible in CA3.

\section{Bursts of action potentials make possible autoassociation in CA3}

Lisman (2003) proposed a mechanism by which an asymmetric learning rule could lead to the symmetrical weight changes required by an autoassociative network. This solution depends on the fact that CA3 neurons often fire brief bursts (maximum within-bursts frequency, $200 \mathrm{~Hz}$; two to five spikes per burst) (Suzuki and Smith 1985). These bursts smear the timing of presynaptic and postsynaptic firing and allow symmetrical weights to be stored. Specifically, at all active cell pairs, some postsynaptic spikes (late in the burst) will occur after a presynaptic spike generated by other CA3 cells early in their burst. When this occurs, the condition for NMDAR-dependent LTP is met (Kampa et al. 2006) bidirectionally between the cells pairs. CA3 can thus store symmetrical weights, even though the learning rule is asymmetric.

\section{Why are CA3 recurrents not random?}

We have argued here that CA3 (at least CA3a) can indeed be considered an autoassociative network. The question remains, however, why the recurrent connections in CA3 (as a whole) should be non-random rather than random. For the purpose of this discussion, we will consider the dentate mossy cells as part of the CA3 system because they share so many properties with CA3 cells: (1) They receive mossy fiber input at large specialized spines near the cell body, (2) they receive CA3 associational inputs on their more distal dendrites, and (3) they receive perforant path input on their most distal dendritic region.

The CA3 system has a huge number of targets (Witter 2007). These include the entire inner third of the molecular layer of the dentate granule cells, the entire ipsilateral and contralateral associational (the recurrent connections) system of CA3, and the feedforward input to CA1, as shown in Figure 6. Given this enormous targeting burden, subdivisions of the CA3 system may have evolved to drive particular targets, at least in a relative way. Thus, mossy cells are specialized to send information back to the dentate (Scharfman 2007); CA3c is specialized to send information to $\mathrm{CA} 1$, and, in the ventral hippocampus, to the dentate (Lee et al.
2004). CA3a is specialized to generate the recurrent associational system. CA3b may simply be intermediate between CA3a and CA3c.

In considering the role of mossy cells and CA3c cells, the following example may be instructive. The input via mossy fibers from the dentate represents memory B, but with certain parts missing (this corruption is indicated as $\mathrm{B}^{\prime}$ ). Thus, some mossy and CA3c cells that are part of memory B will not receive the "detonator" input from mossy fibers. However, the memory will be completed (converted from $\mathrm{B}^{\prime}$ to $\mathrm{B}$ ) when these cells are caused to fire by convergent recurrent inputs from CA3a/b cells that are part of memory B. From this perspective, it can be seen that the fact that mossy and CA3c cells make few recurrent connections is of little functional consequence; what is important is that they receive recurrent synapses.

\section{Storage of the heteroassociative weights required for sequence encoding}

Since the hippocampus stores sequences (Ergorul and Eichenbaum 2006; Foster and Wilson 2006), formation of heteroassociative weights that link sequential items is important. In recent work, we have revised our original idea (Lisman 1999) that heteroassociation occurs in CA3 and now believe that it occurs in the feedback synapses from CA3 to the dentate (Lisman et al. 2005). The key argument that heteroassociation occurs in the dentate is that the passage of information from dentate to CA3 and back to dentate requires $>20 \mathrm{msec}$, producing a delay (see references in Lisman 2005) that turns out to be very important. Provided the next item in the sequence to be learned arrives at the dentate (from cortex) with about the same delay, the inputs will be coincident and will lead to strengthening of the synapses at which the presynaptic activity from CA3 represents the nth item and the active granule cells are driven by the $n+1$ item from cortex. Thus, these synapses will store heteroassociative information, but not autoassociative information. In this way, information that connects different memories is stored at the feedback synapses onto dentate granule cells and can be later used to produce sequence recall (see below).

For this mechanism to work, it is important that the entorhinal cortex not represent sensory information in real time, but rather act as a multiplexing buffer in which information is represented in compressed time (for a definition of compression, see 
Skaggs et al. 1996). According to this view, different "chunks" of sensory information that occurred at substantial temporal separation are represented in cortex within different phases (gamma cycles) of each theta cycle (Lisman and Idiart 1995; Jensen and Lisman 2005), thereby satisfying the temporal requirement (20$30 \mathrm{msec}$ ) for sequential memories described in the previous paragraph. Moreover, each item is actually a "chunk" of information. These ideas have their roots in psychology, particularly in the model presented in Miller (1956). According to this model, the brain has a limited-capacity short-term memory buffer capable of storing $7 \pm 2$ items. Importantly, the definition of an item is not fixed; rather, temporal sequences such as the syllables in a word eventually become represented as a single item representing the word as a whole. Recent work using single-unit recording in humans has found evidence for such high-level representations, with the same unit responding both to a picture of a person and the name of that person (Quiroga et al. 2005). In this way, the seven items in the buffer store information about sequential information that spans considerable time and represents complexity commensurate with the concept of an "episode." In the computational models of such a buffer that we have developed (Jensen and Lisman 1996a,b,c; Jensen et al. 1996), complex items are represented in different gamma cycles, $\sim 30 \mathrm{msec}$ apart, and so can be linked into a sequence by the dentate/CA3 system. We refer the reader to these previous publications for a more detailed description of these models.

\section{Reciprocal connections of CA3 and dentate allow accurate sequence recall and phase precession}

Early theoretical work on the recall of sequences pointed out the necessity for the interaction of autoassociative and heteroassociative weights (Golomb et al. 1990). In the heteroassociative (chaining) step, memory A evokes memory B, but inevitably with small errors (symbolized by $B^{\prime}$ ). If $B^{\prime}$ is used to evoke $C$, the actual version, $\mathrm{C}^{\prime \prime}$, contains even more errors than $\mathrm{B}^{\prime}$. Thus, as chaining occurs during recall, the output gets progressively more corrupted. To avoid this, it was suggested that autoassociative weights be used between each chaining step to correct the representation; a standard capability of autoassociative networks makes it possible to covert a corrupted version of a memory, e.g., $\mathrm{B}^{\prime}$, to its correct form, B.

These requirements for sequence recall can be mapped onto the dentate/CA3 circuitry (Fig. 6), notably the reciprocal connections between dentate and CA3 (Scharfman 2007). The overall process of sequence recall is envisioned as follows (Lisman et al. 2005): Presentation of a cue, A, to the dentate results in activity pattern of this memory being sent to CA3 and back the dentate, where feedback synapses onto granule cells evoked $\mathrm{B}^{\prime}$. This $\mathrm{B}^{\prime}$ is then sent to CA3, where it is corrected to $\mathrm{B}$ and initiates the recall of $C^{\prime}$ in the dentate, and so on. This chaining cycle continues through a theta cycle, but is terminated when theta-mediated inhibition becomes so strong that firing ceases. The recall process must then be reinitiated on the next theta cycle by presentation of a cue.

This model takes on special interest because it provides a simple explanation of the phase precession (O'Keefe and Recce 1993) as a cued recall of a memory sequence. As the rat enters the place field of a cell, firing is initiated but occurs at late theta phase. As the animal progresses through the place field, firing beings progressively earlier on each theta cycle. A simple explanation follows from the idea that a cued sequence chaining process is initiated on each theta cycle. The key point is that the cue is updated at the beginning of each theta cycle. Thus, during the first theta cycle, the cue is position 1 and memories for position $2,3,4$, etc. are evoked during that theta cycle by the chaining process. This is actually a memory-based prediction that the animal will come to these positions. On the second theta cycle, the cue is position 2 (provided the animal moved), so all memories now fire earlier in this theta cycle than they did on the first. A strong prediction of this model is that the phase precession should be linked to how fast the animal is moving (and thus updating cues), and this has been found to be the case (Skaggs et al. 1996). The phase precession is observed in the dentate, CA3, and CA1. According to the above model, it is generated by reciprocal interactions of dentate and CA3, and then presumably passed on to CA1, which is an output structure of the hippocampus.

\section{Phase precession: Why is it present in both the hippocampus and the entorhinal cortex?}

A possible challenge to the above interpretation is posed by recent work showing that phase precession occurs in the grid cells of layer 2 of the entorhinal cortex (Hafting et al. 2007). These are the neurons that provide the input to the dentate/CA3 region. This raises the question of whether it is necessary to invoke the reciprocal interactions between dentate and CA3 to generate the phase precession; perhaps it is simply passed on from cortex.

An important perspective in answering this question relates to the representations used in the cortex and dentate/CA3. It is quite clear that a new representation is formed in dentate granule cells because these receive convergent input from the major subdivision of the entorhinal, the lateral and medial regions. These contain different types of information; notably, information about the animal's position in the environment (grid cells) is evident only in the medial entorhinal cortex (Hargreaves et al. 2005). The lateral entorhinal cortex has strong inputs from inferotemporal cortex and is thus likely to be more sensory driven. In recent theoretical work (Lisman 2007) these ideas were generalized: It was proposed that the medial region carries information about self (including position in space and action taken) whereas the lateral region carries information about the external world, such as landmarks encountered.

As an illustrative example of how information from the lateral and medial inputs might be combined in the dentate/CA3 region, let us consider a sequence of landmark/action couplets (at the light, turn left; at the supermarket, turn right, etc.). Recall of such a stored sequence would allow execution of a complex route to a goal site. Within this context, the lateral entorhinal cortex stores sequences of landmarks, the medial entorinal cortex stores sequences of actions, and the dentate/CA3 stores sequences of landmark/action couplets. Thus, when presented with the cue of a landmark early in a sequence, the lateral region could rapidly (within a theta cycle) recall the upcoming landmarks in the sequence, including the goal, through a chaining process. As the animal moves from one landmark to the next, the cue becomes increasingly later in the sequence; thus, in each successive theta cycle, the excitation of cells that represent a late landmark in the sequence occur earlier and earlier in the chaining process, thereby creating the phase precession in the entorhinal cortex. Importantly, however, this information is not sufficient to navigate to the goal; for this, the associations of landmarks with actions that occur in the dentate/CA3 are necessary. To see how this works, consider what happens when a landmark is seen and this information provides a cue to CA3. There, the autoassociation in CA3 activates the action taken at that landmark; this is the standard pattern completion process that is the hallmark of CA3 computational function. This completed pattern is then sent to the dentate, where the representation of the next landmark/action couplet is evoked through the heteroassociative process. Subsequent reciprocal interactions between dentate and 
CA3 then allow the entire route instructions to be recalled accurately. The phase precession tends to look similar in both cortex and hippocampus, but this is only because it is only positional information that is read out by the experimental protocol; if information about landmarks was also monitored, such information would be represented in the lateral entorhinal, not represented in the medial entorhinal cortex and represented in the dentate and CA3 jointly with positional information.

\section{Conclusion}

The idea that CA3 is an autoassociative network is a longstanding idea. We have considered various challenges to this idea and concluded that none of the objections are fatal. Indeed, with the richness of physiology and anatomy added, one can begin to see how the essential function of autoassociation, as abstracted in nonrealistic Hopfield nets, is executed by real networks. Moreover, the autoassociative process can be placed into the functional context of observable learning and recall processes in the hippocampus.

\section{Acknowledgments}

Marco Idiart and Licurgo de Almeida acknowledge partial financial support from Brazilian agencies CNPq and CAPES.

\section{Appendix}

\section{Storage capacity of the CA3 network}

We follow Jensen et al. (1996) and consider that the synaptic weight between two neurons is always positive and depends on the history of their mutual firing. Since we are interested just in the stable state, we disregard the details of the dynamics of synaptic changes, and assume interleaved learning. In this case the strength of the synaptic connection between two neurons $i$ and $j$ is

$$
W_{i j}=\frac{n_{i j}^{11}}{n_{i j}^{11} \gamma^{11}+n_{i j}^{01} \gamma^{01}+n_{i j}^{10} \gamma^{10}}
$$

where $n_{i j}^{11}$ is the number of patterns where the pre- and postsynaptic neurons $j$ and $i$ fire together, and $n_{i j}^{01}, n_{i j}^{10}$ are defined similarly for non-pre/post and pre/non-post events. The values of $\gamma^{11}=1.40, \gamma^{01}=0.21$, and $\gamma^{10}=0.22$ depend on the specific time constants for AMPA and NMDA channels as indicated in Jensen et al. (1996). The weights in the formula above are zero if the two neurons never fired together and have a modest decrease (LTD) in the case of non-matching firing. For our simulations here the weights varied between 0 and 0.71 .

\section{Integrate-and-fire model}

The pyramidal neurons are modeled as simple, onecompartment, integrate-and-fire neurons; however, important temporal characteristics of membrane processes are considered. The voltage $V_{\mathrm{n}}$ of each neuron if defined by the following equation:

$$
\frac{d V_{\mathrm{n}}}{d t}=\frac{1}{\tau_{\mathrm{n}}}\left(R_{\text {membrane }}\left(I_{\mathrm{ext}}+I_{\mathrm{AHP}}+I_{\mathrm{syn}}+I_{\mathrm{GABA}}\right)-V_{\mathrm{n}}+V_{\text {rest }}\right)
$$

The average input resistance in pyramidal CA3 cells has been found to be $\sim R_{\text {membrane }}=33 \mathrm{M} \Omega$ (Turner and Schwartzkroin 1983), the integration time $\tau_{\mathrm{n}}=2 \mathrm{msec}$, and the rest potential $V_{\text {rest }}=-60 \mathrm{mV}$. A spike is an instantaneous event. When the voltage reaches a threshold $V_{\text {thres }}=-50 \mathrm{mV}$, the cell is reset to $V_{\text {rest }}$.

$I_{\text {ext }}$ can be thought as a memory (or part of this memory) coming from outside CA3. It is a single excitatory stimulus modeled by an alpha function:

$$
I_{\text {ext }}(t)=A_{\text {ext }}\left(\frac{t-t_{\text {ext }}}{\tau_{\text {ext }}} \exp \left(1-\frac{t-t_{\text {ext }}}{\tau_{\text {ext }}}\right)\right)
$$

where $t_{\text {ext }}$ is the time the external neuron fired, $\tau_{\text {ext }}=1.5 \mathrm{msec}$, and $A_{\text {ext }}=480 \mathrm{pA}$.

The after-hyperpolarization current $\left(I_{\mathrm{AHP}}\right)$ prevents pyramidal cells from fast, repetitive firing. $I_{\mathrm{AHP}}$ is modeled here by a decreasing exponential (Jensen et al. 1996):

$$
I_{\mathrm{AHP}}(t)=A_{\mathrm{AHP}} \exp \left(-\frac{t-t_{\text {fire }}}{\tau_{\mathrm{AHP}}}\right)
$$

where $\tau_{\mathrm{AHP}}=5 \mathrm{msec}$ and, for the simulations in this paper, the constant $A_{\mathrm{AHP}}=-560 \mathrm{pA}$.

All synaptic inputs of the recurrent collaterals are excitatory and make one-to-one connections with other pyramidal cells of CA3. The synaptic transmission is mediated by the release of glutamate binding to AMPA and NMDA receptors. In this paper, however, we assume that only AMPA receptors participate in producing EPSP. For this reason, $I_{\text {syn }}$ is basically defined by AMPA parameters and modeled by an alpha function. The synaptic input to cell $i$ is:

$$
\begin{aligned}
I_{\mathrm{syn}}^{i}(t)= & \frac{A_{\mathrm{AMPA}}}{a N} \sum_{j}^{N} W_{i j}\left(\frac{t-t_{\text {fire }}^{j}-t_{\text {delayAMPA }}}{\tau_{\mathrm{AMPA}}}\right) \\
& \exp \left(1-\frac{t-t_{\text {fire }}^{j}-t_{\text {delayAMPA }}}{\tau_{\mathrm{AMPA}}}\right)
\end{aligned}
$$

where $\tau_{\mathrm{AMPA}}=1.5 \mathrm{msec}, A_{\mathrm{AMPA}}=1600 \mathrm{pA}$, and the delay in the recurrent feedback $t_{\text {delayAMPA }}=1.5 \mathrm{msec}$. Here, $t_{\text {fire }}^{j}$ is the action potential of the jth pyramidal cell. The term $a N$ is used to normalize the synaptic input if the network size is changed. $N$ is the number of neurons in our network, and $a$ is a constant representing the sparseness of the memory. That is, $a=A / N$, where $A$ is the number of neurons representing a memory. In this paper we use $A=7$ and $N=30$. The synaptic weight matrix $W_{i j}$ is given by Equation $\mathrm{A} 1$.

The last current acting over our pyramidal neurons is the feedback inhibition. This feedback is responsible for generating gamma oscillations in the following way: The firing of a subset of pyramidal neurons will excite an entire network of inhibitory interneurons through converging excitatory inputs. The interneuron will then provide an inhibitory feedback to all pyramidal cells. After the inhibition wears off, a new subset of pyramidal neurons could become active, and so on. Because we assume all interneurons fire in synchrony, we model the net of GABAergic inputs as:

$$
\begin{aligned}
I_{\mathrm{GABA}}(t)= & \frac{A_{\mathrm{GABA}}}{a N} \sum_{j}^{N}\left(\frac{t-t_{\text {fire }}^{j}-t_{\text {delayGABA }}}{\tau_{\mathrm{GABA}}}\right) \\
& \exp \left(1-\frac{t-t_{\text {fire }}^{j}-t_{\text {delayGABA}}}{\tau_{\mathrm{GABA}}}\right)
\end{aligned}
$$

where, for this paper, $\tau_{\mathrm{GABA}}=4 \mathrm{msec}, A_{\mathrm{GABA}}=-180 \mathrm{pA}$, and $t_{\text {delayAMPA }}=2.5 \mathrm{msec}$.

\section{References}

Abbott, L.F. and Blum, K.I. 1996. Functional significance of long-term potentiation for sequence learning and prediction. Cereb. Cortex 6: 406-416.

Amit, D.J., Gutfreund, H., and Sompolinsky, H. 1987. Information storage in neural networks with low levels of activity. Phys. Rev. A. 35: 2293-2303.

Bains, J.S., Longacher, J.M., and Staley, K.J. 1999. Reciprocal interactions between CA3 network activity and strength of recurrent collateral 
synapses. Nat. Neurosci. 2: 720-726.

Bannister, N.J. and Larkman, A.U. 1995. Dendritic morphology of CA1 pyramidal neurones from the rat hippocampus: II. Spine distributions. J. Comp. Neurol. 360: 161-171.

Bartos, M., Vida, I., and Jonas, P. 2007. Synaptic mechanisms of synchronized gamma oscillations in inhibitory interneuron networks. Nat. Rev. Neurosci. 8: 45-56.

Battaglia, F.P. and Treves, A. 1998. Stable and rapid recurrent processing in realistic autoassociative memories. Neural Comput. 10: 431-450.

Buhmann, J., Divko, R., and Schulten, K. 1989. Associative memory with high information content. Phys. Rev. A. 39: 2689-2692.

Colom, L.V. and Saggau, P. 1994. Spontaneous interictal-like activity originates in multiple areas of the CA2-CA3 region of hippocampal slices. J. Neurophysiol. 71: 1574-1585.

Csicsvari, J., Jamieson, B., Wise, K.D., and Buzsáki, G. 2003. Mechanisms of gamma oscillations in the hippocampus of the behaving rat. Neuron 37: 311-322.

Curti, E., Mongillo, G., La Camera, G., and Amit, D. 2004. Mean field and capacity in realistic network of spiking neurons storing sparsely coded random memories. Neural Comput. 16: 2597-2637.

Dragoi, G. and Buzsáki, G. 2006. Temporal encoding of place sequences by hippocampal cell assemblies. Neuron 50: 145-157.

Dzhala, V.I. and Staley, K.J. 2003. Transition from interictal to ictal activity in limbic networks in vitro. J. Neurosci. 23: 7873-7880.

Ergorul, C. and Eichenbaum, H. 2006. Essential role of the hippocampal formation in rapid learning of higher-order sequential associations. J. Neurosci. 26: 4111-4117.

Foster, D.J. and Wilson, M.A. 2006. Reverse replay of behavioural sequences in hippocampal place cells during the awake state. Nature 440: $680-683$

Frolov, A.A. and Husek, D. 2000. Convergence time in Hopfield network, ijcnn. IEEE-INNS-ENNS International joint conference on neural networks (IJCNN'00), Vol. 5, p. 5622.

Gasparini, S. and Magee, J.C. 2006. State-dependent dendritic computation in hippocampal CA1 pyramidal neurons. J. Neurosci. 26: $2088-2100$.

Gasparini, S., Migliore, M., and Magee, J.C. 2004. On the initiation and propagation of dendritic spikes in CA1 pyramidal neurons. $J$. Neurosci. 24: 11046-11056.

Gold, A.E. and Kesner, R.P. 2005. The role of the CA3 subregion of the dorsal hippocampus in spatial pattern completion in the rat. Hippocampus 15: 808-814.

Golomb, D., Rubin, N., and Sompolinsky, H. 1990. Willshaw model: Associative memory with sparse coding and low fire rates. Phys. Rev. A. 41: $1843-1854$.

Hafting, T., Fyhn, M., Moser, M.B., and Moser, E.I. 2007. Phase precession in entorhinal grid cells. Nature (in press).

Hájos, N., Pálhalmi, J., Mann, E.O., Németh, B., Paulsen, O., and Freund, T.F. 2004. Spike timing of distinct types of GABAergic interneuron during hippocampal gamma oscillations in vitro. $J$. Neurosci. 24: 9127-9137.

Hargreaves, E.L., Rao, G., Lee, I., and Knierim, J.J. 2005. Major dissociation between medial and lateral entorhinal input to dorsal hippocampus. Science 308: 1792-1794.

Henze, D.A., Borhegyi, Z., Csicsvari, J., Mamiya, A., Harris, K.D., and Buzsáki, G. 2000. Intracellular features predicted by extracellular recordings in the hippocampus in vivo. J. Neurophysiol. 84: 390-400.

Hopfield, J.J. 1982. Neural networks and physical systems with emergent collective computational abilities. Proc. Natl. Acad. Sci. 79: 2554-2558.

Ishizuka, N., Weber, J., and Amaral, D.G. 1990. Organization of intrahippocampal projections originating from CA3 pyramidal cells in the rat. J. Comp. Neurol. 295: 580-623.

Jensen, O. and Lisman, J.E. 1996a. Hippocampal CA3 region predicts memory sequences: Accounting for the phase precession of place cells. Learn. Mem. 3: 279-287.

Jensen, O. and Lisman, J.E. 1996b. Novel lists of $7+/-2$ known items can be reliably stored in an oscillatory short-term memory network: Interaction with long-term memory. Learn. Mem. 3: 257-263.

Jensen, O. and Lisman, J.E. 1996c. Theta/gamma networks with slow NMDA channels learn sequences and encode episodic memory: Role of NMDA channels in recall. Learn. Mem. 3: 264-278.

Jensen, O. and Lisman, J.E. 2000. Position reconstruction from an ensemble of hippocampal place cells: Contribution of theta phase coding. J. Neurophysiol. 83: 2602-2609.

Jensen, O. and Lisman, J.E. 2005. Hippocampal sequence-encoding driven by a cortical multi-item working memory buffer. Trends Neurosci. 28: 67-72.

Jensen, O., Idiart, M.A.P., and Lisman, J.E. 1996. Physiological realistic formation of autoassociative memory in networks with theta/gamma oscillations: Role of fast NMDA channels. Learn. Mem. 3: 243-256.

Kampa, B.M., Letzkus, J.J., and Stuart, G.J. 2006. Requirement of dendritic calcium spikes for induction of spike-timing-dependent synaptic plasticity. J. Physiol. 574: 283-290.

Kunec, S., Hasselmo, M.E., and Kopell, N. 2005. Encoding and retrieval in the CA3 region of the hippocampus: A model of theta-phase separation. J. Neurophysiol. 94: 70-82.

Lee, I., Yoganarasimha, D., Rao, G., and Knierim, J.J. 2004. Comparison of population coherence of place cells in hippocampal subfields CA1 and CA3. Nature 430: 456-459.

Leutgeb, S., Leutgeb, J.K., Treves, A., Moser, M.B., and Moser, E. 2004. Distinct ensemble codes in hippocampal areas CA3 and CA1. Science 305: 1295-1298.

Levy, W.B., Sanyal, A., Rodriguez, P., Sullivan, D.W., and Wu, X.B. 2005. The formation of neural codes in the hippocampus: Trace conditioning as a prototypical paradigm for studying the random recoding hypothesis. Biol. Cybern. 92: 409-426.

Li, X.G., Somogyi, P., Ylinen, A., and Buzáki, G. 1994. The hippocampal CA3 network: An in vivo intracellular labeling study. J. Comp. Neurol. 339: 181-208.

Lisman, J. 1999. Relating hippocampal circuitry to function: Recall of memory sequences by reciprocal dentate-CA3 interactions. Neuron 22: $233-242$.

Lisman, J. 2003. Long-term potentiation: Outstanding questions and attempted synthesis. Philos. Trans. R. Soc. Lond. B Biol. Sci. 358: $829-842$.

Lisman, J. 2005. The theta/gamma discrete phase code occurring during the hippocampal phase precession may be a more general brain coding scheme. Hippocampus 15: 913-922.

Lisman, J. 2007. Role of the dual entorhinal inputs to hippocampus: A hypothesis based on cue/action (non-self/self) couplets. Prog. Brain Res. 163: 615-818.

Lisman, J. and Idiart, M.A.P. 1995. Storage of $7 \pm 2$ short-term memories in oscillatory subcycles. Science 267: 1512-1515.

Lisman, J.E., Talamini, L.M., and Raffone, A. 2005. Recall of memory sequences by interaction of the dentate and CA3: A revised model of the phase precession. Neural Netw. 18: 1191-1201.

Malinow, R., Otmakhov, N., Blum, K.I., and Lisman, J. 1994. Visualizing hippocampal synaptic function by optical detection of $\mathrm{Ca}^{2+}$ entry through the N-methyl-D-aspartate channel. Proc. Natl. Acad. Sci. 91: 8170-8174.

Marr, D. 1971. Simple memory: A theory for archicortex. Philos. Trans. R. Soc. Lond. B Biol. Sci. 262: 23-81.

McNaughton, B.L., Battaglia, F.P., Jensen, O., Moser, E.I., and Moser, M.B. 2006. Path integration and the neural basis of the 'cognitive map'. Nat. Rev. Neurosci. 7: 663-678.

Miles, R. 1990. Synaptic excitation of inhibitory cells by single CA3 hippocampal pyramidal cells of the guinea-pig in vitro. J. Physiol. 428: $61-77$.

Miller, C.A. 1956. The magical number seven, plus or minus two: Some limits on our capacity for processing information. Psychol. Rev. 63: 81-97.

Nakazawa, K., Quirk, M.C., Chitwood, R.A., Watanabe, M., Yeckel, M.F., Sun, L.D., Akira Kato, A., Carr, C.A., Johnston, D., Wilson, M.A., et al. 2002. Requirement for hippocampal CA3 NMDA receptors in associative memory recall. Science 297: 211-218.

Oertner, T.G., Sabatini, B.L., Nimchinsky, E.A., and Svoboda, K. 2002. Facilitation at single synapses probed with optical quantal analysis. Nat. Neurosci. 5: 657-664.

O'Keefe, J. and Recce, M.L. 1993. Phase relationship between hippocampal place units and the EEG theta rhythm. Hippocampus 3: 317-330.

Otmakhov, N., Shirke, A.M., and Malinow, R. 1993. Measuring the impact of probabilistic transmission on neuronal output. Neuron 10: 1101-1111.

Pavlidis, P., Montgomery, J., and Madison, D.V. 2000. Presynaptic protein kinase activity supports long-term potentiation at synapses between individual hippocampal neurons. J. Neurosci. 20: $4497-4505$.

Quiroga, R.Q., Reddy, L., Kreiman, G., Koch, C., and Fried, I. 2005. Invariant visual representation by single neurons in human brain. Nature 435: 1102-1107.

Rapp, P.R. and Gallagher, M. 1996. Preserved neuron number in the hippocampus of aged rats with spatial learning deficits. Proc. Natl. Acad. Sci. 93: 9926-9930.

Risau-Gusman S. and Idiart M.A. 2005. Retrieval-time properties of the Little-Hopfield model and their physiological relevance. Phys. Rev. E Stat. Nonlin. Soft Matter Phys. 72: 041913. doi: 10.1103/PhysRevE.72.041913.

Rodriguez, P. and Levy, W.B. 2001. A model of hippocampal activity in trace conditioning: Where's the trace? Behav. Neurosci. 115: $1224-1238$.

Rolls, E.T. and Kesner, R.P. 2006. A computational theory of hippocampal function, and empirical tests of the theory. Prog. 
Neurobiol. 79: 1-48.

Scharfman, H.E. 2007. The CA3 "backprojection" to the dentate gyrus. Prog. Brain Res. 163: 627-637.

Skaggs, W.E., McNaughton, B.L., Wilson, M.A., and Barnes, C.A. 1996. Theta phase precession in hippocampal neuronal populations and compression of temporal sequences. Hippocampus 6: 149-172.

Somogyi, P. and Klausberger, T. 2005. Defined types of cortical interneurone structure space and spike timing in the hippocampus. J. Physiol. 562: 9-26.

Suzuki, S.S. and Smith, G.K. 1985. Burst characteristics of hippocampal complex spike cells in the awake rat. Exp. Neurol. 89: 90-95.

Treves, A. 1993. Mean-field analysis of neural spike dynamics. Network 4: $259-284$.

Treves, A. and Rolls, E. 1991. What determines the capacity of autoassociative memories in the brain? Network 2: 371-397.

Tsodyks, M. and Feigel'man, M. 1988. The enhanced storage capacity in neural networks with low activity level. Europhys. Lett. 6: 101-105.

Turner, D.A. and Schwartzkroin, P.A. 1983. Electrical characteristics of dendrites and dendritic spines in intracellularly-stained CA3 and dentate neurons. J. Neurosci. 3: 2381-2394.

Vazdarjanova, A. and Guzowski, J.F. 2004. Differences in hippocampal neuronal population responses to modifications of an environmental context: Evidence for distinct, yet complementary, functions of CA3 and CA1 ensembles. J. Neurosci. 24: 6489-6496.

Wang, X.J. 1999. Synaptic basis of cortical persistent activity: The importance of NMDA receptors to working memory. J. Neurosci. 19: 9587-9603.

Wang, X.J. 2001. Synaptic reverberation underlying mnemonic persistent activity. Trends Neurosci. 24: 455-463.

Willshaw, D.J., Buneman, O.P., and Longuet-Higgins, H.C. 1969. Non-holographic associative memory. Nature 222: 960-962.

Witter, M. 2007. Intrinsic and extrinsic wiring of CA3, beyond autoassociative network. Learn. Mem. (this issue). doi: $10.1101 / 1 \mathrm{~m} .725207$

Wittner, L., Henze, D.A., Zaborszky, L., and Buzsáki, G. 2006. Hippocampal CA3 pyramidal cells selectively innervate aspiny interneurons. Eur. J. Neurosci. 24: 1286-1298.

Received August 6, 2007; accepted in revised form September 27, 2007. 


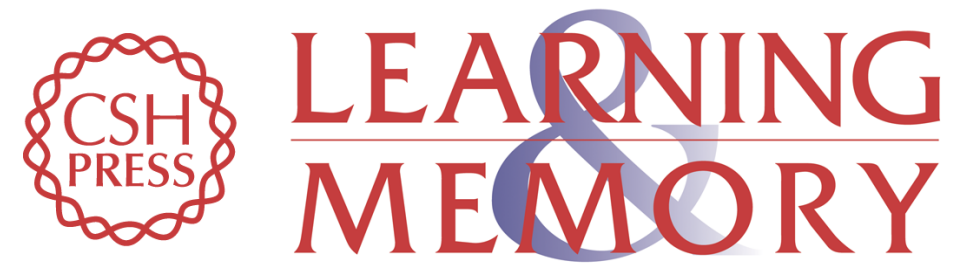

\section{Memory retrieval time and memory capacity of the CA3 network: Role of gamma frequency oscillations}

Licurgo de Almeida, Marco Idiart and John E. Lisman

Learn. Mem. 2007, 14:

Access the most recent version at doi:10.1101//m.730207

References This article cites 70 articles, 21 of which can be accessed free at:

http://learnmem.cshlp.org/content/14/11/795.full.html\#ref-list-1

License

Email Alerting Receive free email alerts when new articles cite this article - sign up in the box at the Service top right corner of the article or click here. 